\title{
A review of the metabolic effects of controlled-release Phentermine/Topiramate
}

\author{
Dimitrios N. Kiortsis
}

Laboratory of Physiology, Medical School, University of Ioannina, Ioannina, Greece

\begin{abstract}
BACKGROUND AND OBJECTIVE: Very few drugs are approved for obesity treatment by regulatory agencies. Very recently phentermine/topiramate controlled-release [PHEN/TPM CR; $\left(\right.$ Qsymia $\left.\left.^{\circledR}\right)\right]$ obtained Food and Drug Administration (FDA) approval as an addition to a reduced-calorie diet and exercise for chronic weight management. Our aim was to review the available clinical evidence on weight loss, metabolic effects and adverse events associated with use of this product. METHODS: Randomized controlled trials with phentermine/topiramate controlled-release were selected through a Medline search using the terms: phentermine and topiramate, phentermine and controlled release topiramate, new anti-obesity drugs and phentermine/topiramate, recent combinations of anti-obesity drugs and Qnexa ${ }^{\circledR}$. RESULTS: PHEN/TPM CR was associated with a weight loss of 8.1-10.9\% (mid and high dose, respectively), while patients in placebo groups lost $1.4-1.8 \%$ of their initial weight. PHEN/TPM CR also resulted in a significant decrease of waist circumference. Weight loss with PHEN/TPM CR was associated with a decrease in blood pressure but with a slight increase in the heart rate. Furthermore, in all trials it exerted favorable effects on lipid profile, especially on triglycerides and high-density lipopoprotein (HDL) cholesterol. PHEN/TPM CR treatment also improved insulin sensitivity and glycemia. Moreover, it decreased significantly progression to type 2 diabetes. In all of the studies the severe adverse events were similar between the control groups and the groups of PHEN/TPM CR. The most frequent side-effects observed in the active treatment group were paresthesia, dysgeusia, dry mouth, constipation and insomnia. WHAT IS NEW AND CONCLUSION: PHEN/TPM CR combined with lifestyle modification may be an effective and well-tolerated treatment for obesity and weight-related metabolic complications. Its long-term efficacy and safety have yet to be defined.
\end{abstract}

Key words: Anti-obesity drugs, Metabolic parameters, Obesity, Phentermine, Topiramate, Weight loss

Address for correspondence:

Professor D.N. Kiortsis, MD, PhD, Laboratory of Physiology, Medical School, University of Ioannina, 45110 Ioannina, Tel.: +3026510 07580, Fax: +3026510 07850,

E-mail: dkiorts@cc.uoi.gr

Received 25-09-2013, Accepted 11-11-2013

\section{BACKGROUND AND OBJECTIVE}

Obesity is a chronic disease which has reached epidemic proportions and is a major public health problem. ${ }^{1}$ It is associated with a wide range of metabolic and cardiovascular conditions such as hyper- 
tension, type 2 diabetes (T2D), hyperlipidemia and atherosclerosis. Obesity is an independent risk factor for cardiovascular disease ${ }^{2,3}$ and predisposes to several types of cancer. ${ }^{4}$ Obesity is also related to other non life-threatening diseases including psychiatric disorders, arthritis and sleep apnea. ${ }^{5}$

Current strategies for the treatment of obesity include lifestyle interventions, pharmacotherapy and bariatric surgery. Although dietary treatment associated with exercise is the cornerstone of treatment, it is very often inadequate. Thus, drug therapy may be important to supplement lifestyle treatment. Antiobesity medication can be classified as centrally-acting or peripherally-acting. ${ }^{6-8}$ Recently, two of the most widely prescribed drugs, sibutramine and rimonabant, were withdrawn from the market. Sibutramine was withdrawn because of an association with increased cardiovascular events and strokes, and rimonabant because of an increased risk of psychiatric adverse events, including depression, anxiety and suicidal ideation. ${ }^{9}$ Previously, other anti-obesity drugs (fenfluramine and dexfenfluramine) were withdrawn due to serious adverse events. Therefore, there is a need for new, more effective and safe drugs. ${ }^{10}$

Drugs approved by the Food and Drug Administration (FDA) for the long-term management of obesity, including weight loss and maintenance of weight loss, are used in conjunction with a reducedcalorie diet and are recommended for patients with an initial body mass index $(\mathrm{BMI}) \geq 30 \mathrm{~kg} / \mathrm{m}^{2}$, or $\geq 27$ $\mathrm{kg} / \mathrm{m}^{2}$ in the presence of other risk factors (e.g., type 2 diabetes (T2D), dyslipidemia, controlled hypertension). The FDA evaluates the efficacy of drugs for obesity primarily based on changes in body weight. According to the 2007 FDA draft guidance for industry entitled "Developing Products for Weight Management," demonstration of efficacy after one year of treatment can be achieved by meeting either of the following co-primary endpoints: (i) the difference in mean weight change (expressed as a percentage change relative to baseline) between the active-treatment group and placebo is $>5 \%$ and the difference is statistically significant; and (ii) more than $35 \%$ of patients lose $\geq 5 \%$ of their baseline body weight in the active-treatment group and this is approximately double the proportion in the placebo group, and the difference in proportions between the active and placebo group is statistically significant.
The respective guidelines of the European Medicines Agency (EMA) state that demonstration of at least $10 \%$ weight loss from baseline weight over one year, which should also be at least 5\% greater than that achieved with placebo, is considered to be a valid primary efficacy criterion in clinical trials evaluating new anti-obesity drugs. In addition, the EMA requires improvements in lipids, glycemia and other obesityrelated metabolic abnormalities.

Recently, two new drugs have been approved by the FDA for the management of obesity: Lorcaserin $\left(\right.$ Belviq $\left.^{\circledast}\right)$ and phentermine/topiramate controlledrelease (PHEN/TPM CR) $\left(\right.$ Qsymia $\left.^{\circledR}\right)$. In the present review we discuss the efficacy and the metabolic effects as well as the adverse events characterizing the new anti-obesity drug PHEN/TPM CR (Qsymia ${ }^{\circledR}$ ) (the original name was Qnexa ${ }^{\circledR}$ ).

\section{METHODS}

Relevant articles were identified through a Medline search (up to October 2013) using different terms as "phentermine and topiramate, phentermine and controlled release topiramate, phentermine in combination with topiramate, new anti-obesity drugs and phentermine/topiramate, recent combinations of anti-obesity drugs, phentermine/topiramate and review, and Qnexa ${ }^{\circledR}$ ". We focused on papers with larger numbers of patients and with key observations related to the topic of this review.

\section{PHENTERMINE (PHEN)}

Phentermine was approved for use by the FDA in 1959 as a short-term (<12 weeks) treatment for weight loss in combination with appropriate nutrition and physical exercise (recommended dose $37.5 \mathrm{mg} /$ day). ${ }^{11,12}$ It is available in some countries but not in Europe, where it was withdrawn in 2000 due to an unfavorable risk to benefits ratio. ${ }^{13}$ Phentermine is an indirect-acting sympathomimetic agent that acts by releasing noradrenaline (NA) from presynaptic vesicles in the lateral hypothalamus. The increase in NA concentration within the synaptic cleft results in the stimulation of $\beta 2$-adrenergic receptors and a resultant suppression. ${ }^{14,15}$ Some evidence suggests that its weight loss effects are mainly due to increase in 
resting energy expenditure. ${ }^{11}$ It is chemically related to amphetamine but does not have its addictive potential. Phentermine is listed as a Schedule IV drug because it has a low abuse potential. ${ }^{11}$ In contrast, amphetamine is listed as a Schedule II drug. ${ }^{11}$

Phentermine has been evaluated as both monotherapy and as combination therapy.${ }^{14} \mathrm{~A}$ meta-analysis assessed randomized controlled trials (RCTs) of the use of phentermine for weight loss in obese patients. ${ }^{16}$ Nine studies, published between 1975 and 1999, were included. According to this meta-analysis, subjects treated with phentermine lost an average of 3.6 additional $\mathrm{kg}$ of weight compared to placebo $(95 \% \mathrm{CI}$, 0.6 to 6.0 ). The duration of treatment varied from 2 to 24 weeks. Moreover, patients treated with phentermine maintained "fairly substantial" weight loss compared with placebo $(2.43 \mathrm{~kg})$ after discontinuation of the drug.

Phentermine is contraindicated when sympathomimetic drug administration may pose a significant risk, such as in patients with unstable cardiovascular disease, moderate-to-severe high BP, hyperthyroidism and unstable cardiac dysrhythmias. Phentermine is not recommended in patients treated with monoamine oxidase inhibitors or in patients with a history of drug abuse (including excessive alcohol consumption). Phentermine should be used with caution in patients concurrently treated with other agents that affect the Central Nervous System (CNS), such as drugs which increase adrenergic responses, i.e. decongestants. The most common side-effects of phentermine are tachycardia, increase in blood pressure (BP), anxiety, dizziness, insomnia, headache, dryness of the mouth and gastrointestinal complaints. ${ }^{12,17,18}$

\section{TOPIRAMATE (TPM)}

Topiramate is an anticonvulsant approved in the mid-1990s for the treatment of refractory seizures (recommended dose $400 \mathrm{mg} /$ day) in conjunction with other anticonvulsants. It can also be used for prevention of migraine headaches (recommended dose 100 $\mathrm{mg} /$ day). ${ }^{17}$ Topiramate is a sulfamate-substituted monosaccharide derivative of the naturally occurring sugar monosaccharide d-fructose.${ }^{11}$ In the process of testing topiramate for treatment of mood disorders, it was discovered that the agent might mitigate the weight gain often observed with antidepressant treatment, and a dose-ranging study has established that these effects are dose-dependent. ${ }^{12,17}$ This explains why some clinicians have used topiramate "off label" for the purpose of weight reduction, for which it has no regulatory approval.

The mechanisms for topiramate's weight loss effect have not been fully elucidated. Topiramate's antagonism of a-amino-3-hydroxy-5-methyl-4-isoxazole-propionic acid kainate (AMPA/KA) receptors may reduce compulsive or addictive food cravings, ${ }^{19}$ which is supported by topiramate's effectiveness in improving binge eating disorder ${ }^{20}$ and in reducing other addictive behaviors. ${ }^{21}$ Topiramate's activation of gamma-aminobutyric acid (GABA) receptors is likely to decrease night-time and deprivation-induced feeding, ${ }^{22}$ which results in persistent weight loss in combination with decreased feeding. While animal studies suggest that topiramate could increase the levels of neuropeptide Y (NPY) in the hypothalamus (an effect that would seem to promote positive caloric balance, ${ }^{2}$ ) topiramate might also increase levels of hypothalamic corticotropic-releasing hormone, which are likely catabolic. ${ }^{24}$ It may also increase hypothalamic galanin, with unclear body weight implications. While some studies suggest leptin levels decrease with topiramate-induced weight reduction, ${ }^{25}$ other reports indicate that leptin levels are either not significantly decreased, or only modestly decreased with topiramate-induced weight loss, which is another effect that may favor persistence of weight loss. ${ }^{26}$ Anorexia is commonly reported with topiramate; however, topiramate probably does not reduce objective measures of appetite during weight loss. Reduced hunger and appetite during weight loss could reflect cumulative anorectic effects of topiramate, balanced against the orexigenic effects of topiramate-promoted fat loss or related to energy deficit through other mechanisms. ${ }^{27}$ This would favor persistence of weight loss. Regarding energy expenditure, topiramate might decrease energy storage and usage efficiency and thus increase energy expenditure. ${ }^{27}$ Topiramate might inhibit adipocyte mitochondrial carbonic anhydrase isozyme $\mathrm{V},{ }^{28}$ thereby inhibiting carbonic anhydrase-mediated lipogenesis. ${ }^{29,30}$ Topiramate probably also affects lipoprotein lipase activity in white and brown adipose tissue, which would limit free fatty acid substrate for lipogenesis and may increase thermogenesis. ${ }^{31}$ 
An interesting meta-analysis of six studies assessed the efficacy of topiramate in terms of weight loss (Figure 1). ${ }^{12}$ The pooled random-effects estimate of the percent weight loss in topiramate-treated patients compared with placebo-treated patients was $6.5 \%$ ( $95 \%$ CI, $4.8 \%$ to $8.3 \%$ ) for 24 weeks of treatment. In a dose comparison, topiramate at $192 \mathrm{mg} /$ day produced more weight loss than $96 \mathrm{mg} /$ day.

Topiramate may have adverse effects, many of them being dose-dependent. Some of them could be related to carbonic anhydrase inhibition, such as paresthesia, dysgeusia, metabolic acidosis, increased risk of kidney stones and oligohidrosis. Other sideeffects are independent of the inhibition of carbonic anhydrase, such as anorexia, fatigue and somnolence. Moreover, other adverse events have been reported such as dry mouth, constipation and, less commonly, diarrhoea. Special mention should be made of the potential CNS adverse experience of topiramate, although rare, which includes depression and difficulty with concentration and memory. Finally, topiramate can very infrequently cause acute myopia, sudden onset of blurring of vision, redness of the sclera, photophobia and discomfort of the eyes resulting from secondary angle-closure glaucoma. ${ }^{11,12,17}$

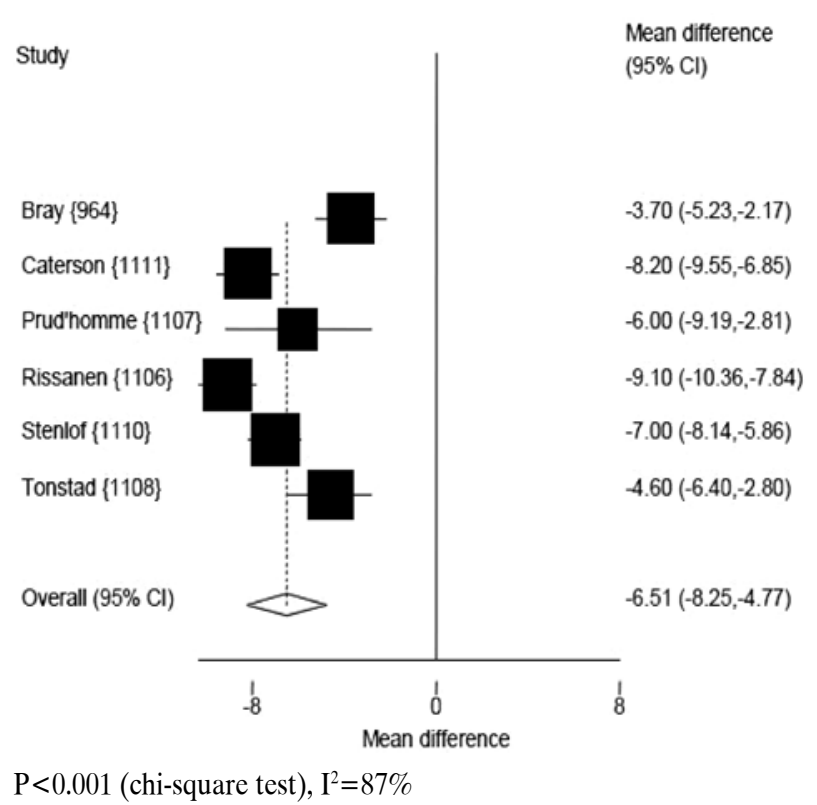

Figure 1. Meta-analysis of six studies of weight loss effects of topiramate.

\section{PHENTERMINE/TOPIRAMATE CR (QSYMIA ${ }^{\circledR}$ )}

After the withdrawal of sibutramine and rimonabant, the need for new, safe and effective anti-obesity drugs became urgent. Qsymia ${ }^{\circledR}$, a combination drug which consists of low doses of immediate-release phentermine and controlled-release topiramate, was approved by the US FDA in July 2012 as an addition to a reduced-calorie diet and exercise for chronic weight management. The combination is being assessed for this indication by the EMA. The effectiveness and safety of Qsymia ${ }^{\circledR}$ has been evaluated in several Phase III trials (Table 1)..$^{32-34}$

EQUATE (Vivus inc press release Dec. $11^{\text {th }}$ 2008) was a 28 -week study. It included 756 patients, $79 \%$ of whom were women with a BMI $=30-45 \mathrm{~kg} / \mathrm{m} .{ }^{2}$ The treatments used were placebo, phentermine $7.5 \mathrm{mg}$, phentermine $15 \mathrm{mg}$, topiramate CR $46 \mathrm{mg}$, topiramate CR 92mg, PHEN/TPM CR 7.5/46mg and PHEN/TPM CR $15 / 92 \mathrm{mg}$. The weight loss was $1.7 \%$ with placebo, almost $6 \%$ with phentermine or topiramate and about $9 \%$ with the combination treatment $(p<0.001)$. The proportion of patients with PHEN/TPM CR who achieved weight loss of over $5 \%$ of their initial body weight was more than triple that observed with placebo. The percentage of patients who lost more than $10 \%$ of their initial body weight was $41 \%, 39 \%$ and $7 \%$, for PHEN/TPM CR full, mid dose and placebo, respectively. The dropout rate was about $30 \%$.

EQUIP started in November 2007 and was completed in May 2009. It included 1267 men and women (aged 18-70 years) with class II and III obesity (BMI $\geq 35 \mathrm{~kg} / \mathrm{m}^{2}$ ), fasting blood glucose $(\mathrm{FBG})<110 \mathrm{mg} / \mathrm{dl}$, triglycerides $(\mathrm{TG}) \leq 200 \mathrm{mg} / \mathrm{dl}$ and $\mathrm{BP} \leq 140 / 90 \mathrm{mmHg}$ (patients could be on medication for TG and BP). They were randomized to placebo $(\mathrm{n}=514), \mathrm{PHEN} / \mathrm{TPM}$ CR 3.75/23mg ( $\mathrm{n}=241)$, or PHEN/TPM CR 15/92mg $(\mathrm{n}=512)$, added to a reduced-energy diet. Patients lost $1.6 \%, 5.1 \%$ and $10.9 \%$ of baseline body weight, respectively, at 56 weeks $(p<0.0001)$. Percentages of patients who lost $\geq 5 \%$ of body weight were $66.7 \%$ on $15 / 92,44.9 \%$ on $3.75 / 23$ and $17.3 \%$ on placebo. Statistically significantly more patients achieved $10 \%$ and $15 \%$ weight loss in both active treatment groups compared with the placebo group.

CONQUER was a 56-week study and included 2487 patients (aged 18-70 years) with a BMI $=27-45$ 


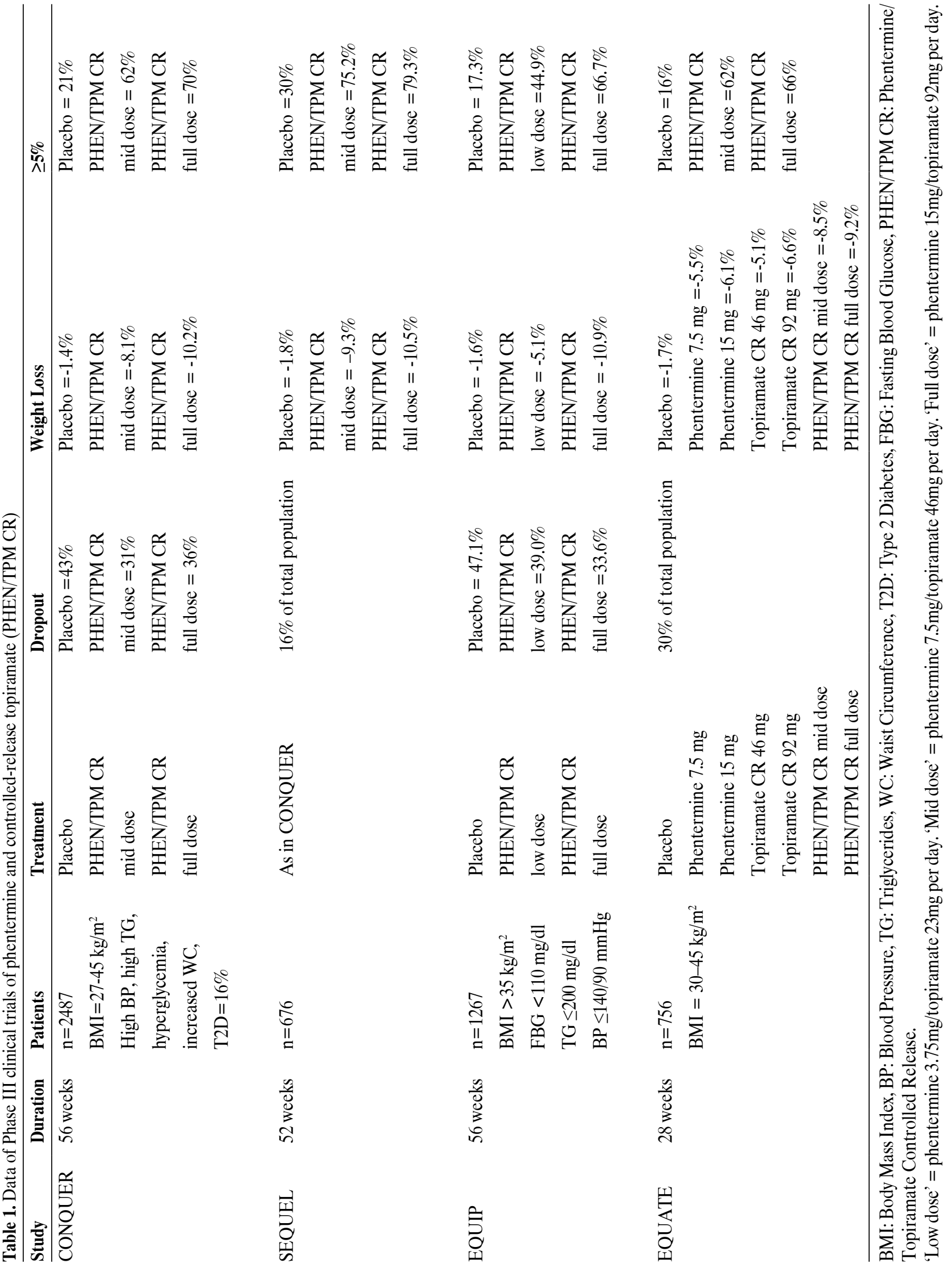


$\mathrm{kg} / \mathrm{m}^{2}$ and two or more of the following co-morbidities: systolic BP $(\mathrm{SBP})=140-160 \mathrm{mmHg}$, diastolic BP $(\mathrm{DBP})=90-100 \mathrm{mmHg}, \mathrm{TG}=2.26-4.52 \mathrm{mmol} / \mathrm{L}, \mathrm{FBG}$ $>5.55 \mathrm{mmol} / \mathrm{L}$, increased waist circumference (WC) or T2D (patients could be on medication for BP, TG and diabetes). The patients were assigned either to placebo $(\mathrm{n}=944)$, PHEN/TPM CR 7.5/46mg $(\mathrm{n}=498)$ or PHEN/TPM CR 15/92mg ( $\mathrm{n}=995)$ and the change in body weight was $-1.4 \mathrm{~kg},-8.1 \mathrm{~kg}$ and $-10.2 \mathrm{~kg}$, respectively $(p<0.0001)$. The $21 \%$ of the patients who were on placebo achieved at least $5 \%$ weight loss, the $62 \%$ on PHEN/TPM CR $7.5 / 46 \mathrm{mg}$ and the $70 \%$ on PHEN/TPM CR 15/92mg; for $\geq 10 \%$ weight loss, the corresponding numbers were $7 \%, 37 \%$ and $48 \%$.

Interestingly, in EQUIP and in CONQUER more patients discontinued treatment in the placebo group compared to the PHEN/TPM CR groups. A possible explanation is that patients in the active treatment groups observed better results mainly in terms of weight loss and they were therefore encouraged to continue their treatment.

SEQUEL, a 52-week extension of the CONQUER study, started in December 2008 and was completed in June 2010. The participants $(n=676)$ continued the original treatment to which they were randomly assigned during CONQUER. Least-squares mean percentage changes from baseline in body weight were $-1.8 \%,-9.3 \%$ and $-10.5 \%$ for placebo, PHEN/TPM CR 7.5/46 and PHEN/TPM CR 15/92, respectively.
Significantly more PHEN/TPM CR-treated patients at each dose achieved $\geq 5 \%, \geq 10 \%, \geq 15 \%$ and $\geq 20 \%$ weight loss compared with placebo $(p<0.001)$. The completion rate was about $84 \%$.

\section{EFFECTS ON METABOLIC AND OTHER PARAMETERS}

In the large randomized trials, significant improvements in several metabolic and other obesity-related co-morbidities were observed (Table 2). ${ }^{32-34}$

Waist circumference (WC) decreased significantly in all active treatment groups in all studies, but more markedly in the full-dose groups. Specifically, there was a decrease in WC from 9.2 to $10.9 \mathrm{~cm}$ in the fulldose groups, while in the placebo groups there was a reduction from 2.4 to $3.6 \mathrm{~cm}$.

\section{Effects on blood pressure}

In all the studies, systolic and diastolic blood pressure reduced significantly from baseline in PHEN/ TPM CR groups, especially with the full dose, while in the groups taking placebo it decreased by a lower percentage or even increased. Of note, in most trials the decrease in blood pressure in the PHEN/TPM CR groups was statistically significantly different from that observed in the placebo arms. The placebo effect was greater in SEQUEL than in the other two trials. In the latter study there was no significant difference in blood pressure effect between treatment

Table 2. Mean changes from baseline of metabolic and other parameters with phentermine and controlled-release topiramate (PHEN/TPM CR) treatment and placebo

\begin{tabular}{|c|c|c|c|c|}
\hline \multicolumn{2}{|l|}{ Metabolic Parameter } & \multirow{2}{*}{$\begin{array}{c}\text { Placebo } \\
-3.2 \text { to } 0.9\end{array}$} & \multirow{2}{*}{$\begin{array}{c}\text { Low Or Mid Dose } \\
-4.7 \text { to }-1.8\end{array}$} & \multirow{2}{*}{$\begin{array}{c}\text { Full Dose } \\
-5.6 \text { to }-2.9\end{array}$} \\
\hline Blood pressure (mm Hg) & SBP & & & \\
\hline & DBP & -3.9 to 0.4 & -3.7 to -0.1 & -3.8 to -1.5 \\
\hline FBG (mmol/L) & & 0.13 to 3.7 & -0.01 to 0.8 & -1.2 to -0.07 \\
\hline Glycated hemoglobin (\%) & & 0.1 to 0.2 & 0 to 0.01 & -0.1 to 0 \\
\hline $\mathrm{TG}(\%)$ & & 0.4 to 9.1 & -12.5 to -8.6 & -13.7 to -5.2 \\
\hline LDL cholesterol $(\%)$ & & -10.7 to -4.1 & -7.7 to -3.7 & -8.4 to -5.6 \\
\hline HDL cholesterol (\%) & & 0 to 4.7 & 5.2 to 7.3 & 3.5 to 11.9 \\
\hline Total cholesterol (\%) & & -3.5 to -3.3 & -5.4 to -4.9 & -6.3 to -6 \\
\hline Waist circumference $(\mathrm{cm})$ & & -3.6 to -2.4 & -9.8 to -5.6 & -10.9 to -9.2 \\
\hline
\end{tabular}

All the changes were statistically significant between active treatment and placebo except for LDL-C and in some trials for Blood Pressure, mainly DBP. SBP: Systolic Blood Pressure, DBP: Diastolic Blood Pressure, FBG: Fasting Blood Glucose, TG: Triglycerides, LDL: Low Density Lipoprotein, HDL: High Density Lipoprotein. 
arms. ${ }^{33}$ This may have been due to a net increase in the number of antihypertensive medications used by the placebo group, whereas there was a decrease in the number of medications in subjects receiving both doses of active treatment.

\section{Effects on glycemic control and type 2 diabetes}

The statistically significant reduction in fasting blood glucose was seen mainly with the 15/92 dose. Moreover, in the active treatment groups an improvement in glycated hemoglobin was observed compared to placebo. More patients in the placebo groups needed an increase in the number of antidiabetic drugs than in the PHEN/TPM CR groups. Notably, patients with both doses of phentermine plus topiramate and with diabetes at baseline exhibited more pronounced reductions in glycated hemoglobin than the overall sample. ${ }^{32,33}$ These results suggest that the treatment may be more beneficial for diabetic subjects. SEQUEL showed that in subjects without T2D at baseline, the favorable effects of weight loss on insulin sensitivity and glycemia were associated with decreased progression to T2D during the $2^{\text {nd }}$ year of study. A 54\% reduction in progression to T2D in subjects taking 7.5/46 and a $76 \%$ reduction in subjects receiving the $15 / 92$ compared to placebo were observed. ${ }^{33}$ Interestingly, in a very recent study in patients with metabolic syndrome and/or prediabetes, treatment with PHEN/TPM CR resulted in a $70 \%$ reduction in progression to $\mathrm{T} 2 \mathrm{D}$ in those taking $7.5 / 46$ and a $79 \%$ reduction in those receiving the $15 / 92$ compared to placebo. ${ }^{35}$ In the several studies, no assessment of the effects of treatment on metabolic syndrome and on non-alcoholic fatty liver disease (NAFLD) was performed. However, the impressive weight loss achieved with PHEN/TPM CR as well as the significant improvement of the metabolic profile may suggest that more than $50-60 \%$ of patients would no longer present these two conditions after treatment.

\section{Effects on lipids}

In all studies, treatment with PHEN/TPM CR led to greater reductions in triglycerides (TG) and greater increases in high-density lipoprotein (HDL) cholesterol than did placebo, especially with the full dose. ${ }^{32-34} \mathrm{~A}$ decrease in low-density lipoprotein (LDL) cholesterol from baseline was observed with both PHEN/TPM CR and placebo. In most studies, reductions in LDL-cholesterol were not statistically more pronounced in the active treatment groups than in the placebo groups. Only in SEQUEL did the placebo group experience a greater decrease in LDL-cholesterol than in the PHEN/TPM CR groups. ${ }^{33}$ However, during this study more patients in the PHEN/TPM CR groups decreased their consumption of lipid-lowering drugs. In most studies, more patients in the placebo group needed an increase in lipid-lowering medication compared to patients in the PHEN/TPM CR groups.

\section{ADVERSE EVENTS}

In all the studies, the severe adverse events were similar between the PHEN/TPM CR groups and the placebo groups. ${ }^{32-34}$ Most of the common adverse events could be interpreted in relation to what is known about the adverse effects of the two drugs individually. In all the studies no statistically significant changes in heart rate were observed in patients on low and mid doses compared to placebo. ${ }^{32-34}$ Patients who received full doses experienced a small increase in mean heart rate of 1.2-1.7beats/min, an effect which was statistically significantly different from the changes observed in the placebo groups in two trials ${ }^{32,33}$ but not in a third trial. ${ }^{34}$ The most frequent side-effects were paresthesia, dysgeusia, dry mouth, constipation and insomnia. ${ }^{11,32-34}$ These adverse events occurred in patients who received full and mid doses but not in those who took the low dose. Irritability, anxiety, disturbance in attention and nephrolithiasis were less common side-effects observed in EQUIP and CONQUER in subjects receiving either full or mid doses. Dry eye, dry skin, decreased serum bicarbonate and feeling jittery were seen in CONQUER but only in the 15/92 group. In SEQUEL, which assessed the safety and tolerability of PHEN/TPM CR for 108 weeks, the adverse events which occurred between weeks 54 and 108 were similar to those reported in the other trials in the first year of treatment. However, the incidence was markedly lower in the second year than in the first year. ${ }^{33}$

Several scientists and the FDA raised the issue of potential birth defects associated with PHEN/TPM $\mathrm{CR}$ treatment. These concerns were based on a few studies in animals and humans which assessed the po- 
tential teratogenic effect of topiramate. ${ }^{36-38}$ The results of clinical studies assessing the role of PHEN/TPM CR treatment on birth defects are inconclusive. In EQUIP there were 15 pregnancies in women exposed to PHEN/TPM CR. ${ }^{34}$ Among these, there were three spontaneous abortions, three elective abortions and nine healthy live births, while no congenital malformations were observed. During SEQUEL, there were two pregnancies, one carried to term in the $15 / 92$ group and one resulting in miscarriage at approximately 6 wk gestation in the placebo group. ${ }^{33}$ The pregnancy that was carried to full term resulted in a healthy macrosomic infant with no observed teratogenic effects. Accordingly, the FDA approved Qsymia ${ }^{\circledR}$ with a Risk Evaluation and Mitigation Strategy (REMS), which consists of a Medication Guide providing patients with important safety information that includes prescriber training and pharmacy certification. The purpose of the REMS is to educate prescribers and their patients about the increased risk of birth defects associated with first trimester exposure to Qsymia ${ }^{\circledR}$, the need for pregnancy prevention and the need to discontinue therapy if pregnancy occurs. Qsymia ${ }^{\circledR}$ will only be dispensed through specially certified pharmacies. Depression is a major concern connected with several anti-obesity drugs and in EQUIP it occurred at a higher frequency only in patients who received PHEN/TPM CR 15/92. In CONQUER, although the incidence of depression was higher with the full dose than with placebo, various analyses of Patient Health Questionnaire (PHQ-9) scores showed no differences between the three groups and the use of new antidepressant drugs by the patients did not increase. Neither suicidal behavior nor suicidal ideation with intent to act was reported in the different studies. Importantly, after two years of treatment (SEQUEL trial), the occurrence of depression-related adverse events were comparable in the placebo and the 15/92 group. Thus, PHEN/TPM CR at the full dose may increase depression mainly during the early phase of treatment. In the large clinical trials, participation of patients with a history of depression, with adequately controlled depression based on PHQ-9 scores, and those on stable doses of antidepressants was allowed. The main exclusion criteria were recurrent major depression, presence or history of suicidal behavior or ideation with intent to act and current substantial depressive symptoms. Therefore, probably treatment with PHEN/TPM CR should be avoided by patients with uncontrolled depression or with suicidal behavior or ideation with intent to act.

\section{LIMITATIONS OF STUDIES}

The limitations of these large trials were primarily the overrepresentation of women (70-80\%) and the lack of ethnic diversity ( $\sim 80 \%$ white persons). ${ }^{16,33}$ In CONQUER as well as in SEQUEL, there was a restriction on the upper limit of BMI to $45 \mathrm{~kg} / \mathrm{m}^{2}$, while in EQUIP many participants did not have any significant obesity-associated co-morbid diseases.

\section{WHAT IS NEW AND CONCLUSION}

PHEN/TPM CR treatment with lifestyle interventions produces significant weight loss compared to placebo. Moreover, an improvement in the metabolic profile was observed. The efficacy of PHEN/TPM CR seems very favorable compared with the efficacy of existing and other emerging drugs. Placebo-subtracted weight loss with PHEN/TPM CR was between $6.5 \%$ and $8.6 \%$, while with orlistat it was less than $3 \%$ and with lorcaserin, the other anti-obesity drug newly approved by the FDA, 3.3\%. However, randomized head-to-head trials are needed in order to draw any definite conclusions about relative efficacy of the various drugs. ${ }^{39,40} \mathrm{PHEN} / \mathrm{TPM} \mathrm{CR}$ was well-tolerated and there was no increase in severe adverse events compared to placebo. Moreover, though the cost of this drug is quite high, it is similar to that of other anti-obesity drugs currently or previously on the market. Given that the cost of obesity and obesityrelated diseases is substantial, the cost-benefit ratio of long-term PHEN/TPM CR treatment should be assessed.

Further studies with larger groups of men and people of various ethnic origins will surely be informative. In future studies, determining the extent to which weight loss is maintained in the longer term would be useful. Moreover, further trials are needed in order to assess the long-term safety of PHEN/ TPM CR treatment beyond two years in view of the fact that obesity is a relapsing disease and chronic pharmacotherapy may be needed. 


\section{REFERENCES}

1. Flegal KM, Carroll MD, Ogden CK, Johnson CL, 2002 Prevalence and trends in obesity among US adults, 1999-2000. J Am Med Assoc 288: 1723-1727.

2. Klein S, Burke LE, Bray GA, et al, 2004 Clinical implications of obesity with specific focus on cardiovascular disease: a statement for professionals from the American Heart Association Council on Nutrition, Physical Activity, and Metabolism: endorsed by the American College of Cardiology Foundation. Circulation 110: 2952-2967.

3. Poirier P, Giles TD, Bray GA, et al, 2006 Obesity and cardiovascular disease: pathophysiology, evaluation, and effect of weight loss: an update of the 1997 American Heart Association Scientific Statement on Obesity and Heart Disease from the Obesity Committee of the Council on Nutrition, Physical Activity, and Metabolism. Circulation 113: 898-918.

4. Heal DJ, Gosden J, Smith SL, 2009 Regulatory challenges for new drugs to treat obesity and comorbid metabolic disorders. Br J Clin Pharmacol 68: 861-874.

5. Pi-Sunyer FX, 2004 The epidemiology of central fat distribution in relation to disease. Nutr Rev 62: 120-126.

6. Kiortsis DN, Filippatos TD, Elisaf MS, 2005 The effects of orlistat on metabolic parameters and other cardiovascular risk factors. Diabet Metab 31: 15-22.

7. Filippatos TD, Kiortsis DN, Liberopoulos EN, Mikhailidis DP, Elisaf MS, 2005 A review of the metabolic effects of sibutramine. Curr Med Res Op 21: 457-466.

8. Christopoulou FD, Kiortsis DN, 2011 An overview of the metabolic effects of rimonabant in randomized controlled trials: potential for other cannabinoid 1 receptor blockers in obesity. J Clin Pharm Ther 36: 10-18.

9. Kang JG, Park CY, 2012 Anti-Obesity Drugs: A Review about Their Effects and Safety. Diabetes Metab J 36: 13-25.

10. Klonoff DC, Greenway F, 2008 Drugs in the pipeline for the obesity market. J Diabetes Sci Technol 2: 913-918.

11. Bays H, 2010 Phentermine, topiramate and their combination for the treatment of adiposopathy ('sick fat') and metabolic disease. Expert Rev Cardiovasc Ther 8: 1777-1801.

12. Li Z, Maglione M, Tu W, et al, 2005 Meta-analysis: pharmacologic treatment of obesity. Ann Intern Med 142:532-546.

13. Glazer G, 2001 Long-term pharmacotherapy of obesity 2000: a review of efficacy and safety. Arch Intern Med 161: 1814-1824.

14. Ioannides-Demos LL, Piccenna L, McNeil JJ, 2011 Pharmacotherapies for obesity: past, current, and future therapies. J Obes 2011: 179674.

15. Witkamp RF, 2011 Current and future drug targets in weight management. Pharm Res 28: 1792-1818.

16. Haddock CK, Poston WS, Dill PL, Foreyt JP, Ericsson M, 2002 Pharmacotherapy for obesity: a quantitative analysis of four decades of published randomized clinical trials. Int J Obes Relat Metab Disord 26: 262-273.
17. Shekelle PG, Morton SC, Maglione M, et al, 2004 Pharmacological and surgical treatment of obesity. Evid Rep Technol Assess 103: 1-6.

18. Kennett GA, Clifton PG, 2010 New approaches to the pharmacological treatment of obesity: can they break through the efficacy barrier? Pharmacol Biochem Behav 97: 63-83.

19. Khazaal Y, Zullino DF, 2007 Topiramate-induced weight loss is possibly due to the blockade of conditioned and automatic processes. Eur J Clin Pharmacol, 63: 891-892.

20. McElroy SL, Arnold LM, Shapira NA, et al, 2003 Topiramate in the treatment of binge eating disorder associated with obesity: a randomized, placebo-controlledtrial. Am J Psychiatry 160: 255-261.

21. Khazaal Y, Zullino DF, 2006 Topiramate in the treatment of compulsive sexual behavior: case report. BMC Psychiatry 6: 22.

22. Turenius CI, Htut MM, Prodon DA, et al, 2009 GABA(A) receptors in the lateral hypothalamus as mediators of satiety and body weight regulation. Brain Res 1262: 16-24.

23. Erondu N, Gantz I, Musser B, et al, 2006 Neuropeptide $\mathrm{Y} 5$ receptor antagonism does not induce clinically meaningful weight loss in overweight and obese adults. Cell Metab 4: 275-282.

24. Husum H, Van Kammen D, Termeer E, Bolwig G, Mathé A, 2003 Topiramate normalizes hippocampal NPY-LI in flinders sensitive line 'depressed' rats and upregulates NPY, galanin, and CRH-LI in the hypothalamus: implications for mood-stabilizing and weight loss-inducing effects. Neuropsychopharmacology 28: 1292-1299.

25. Schütt M, Brinkhoff J, Drenckhan M, Lehnert H, Sommer C, 2010 Weight reducing and metabolic effects of topiramate in patients with migraine--an observational study. Exp Clin Endocrinol Diabetes 118: 449-452.

26. Theisen FM, Beyenburg S, Gebhardt S, et al, 2008 A prospective study of body weight and serum leptin levels in patients treated with topiramate. Clin Neuropharmacol 31: 226-230.

27. Tremblay A, Chaput JP, Bérubé-Parent S, et al, 2007 The effect of topiramate on energy balance in obese men: a 6-month double-blind randomized placebo-controlled study with a 6-month open-label extension. Eur J Clin Pharmacol 63: 123-134.

28. Winum JY, Scozzafava A, Montero JL, Supuran CT, 2005 Sulfamates and their therapeutic potential. Med Res Rev 25: 186-228.

29. Poulsen SA, Wilkinson BL, Innocenti A, Vullo D, Supuran CT, 2008 Inhibition of human mitochondrial carbonic anhydrases VA and VB with para-(4-phenyltriazole1-yl)-benzenesulfonamide derivatives. Bioorg Med Chem Lett 18: 4624-4627.

30. Vullo D, Franchi M, Gallori E, Antel J, Scozzafava A, Supuran CT, 2004 Carbonic anhydrase inhibitors. Inhibition of mitochondrial isozyme $\mathrm{V}$ with aromatic and heterocyclicsulfonamides. J Med Chem 47: 1272-1279. 31. Richard D, Ferland J, Lalonde J, Samson P, Deshaies Y, 
2000 Influence of topiramate in the regulation of energy balance. Nutrition 16: 961-966.

32. Gadde KM, Allison DB, Ryan DH, et al, 2011 Effects of low dose, controlled release, phentermine plus topiramate combination on weight and associated comorbidities in overweight and obese adults (CONQUER): a randomised, placebo-controlled, phase 3 trial. Lancet 377: 1341-1352.

33. Garvey WT, Ryan DH, Look M, et al, 2012 Two-year sustained weight loss and metabolic benefits with controlledrelease phentermine/topiramate in obese and overweight adults (SEQUEL): a randomized, placebo-controlled, phase 3 extension study. Am J Clin Nutr 95: 297-308.

34. Allison DB, Gadde KM, Garvey WT, et al, 2012 Controlled-release phentermine/topiramate in severely obese adults: a randomized controlled trial (EQUIP). Obesity (Silver Spring) 20: 330-342.

35. Garvey WT, Ryan DH, Henry R, et al, 2013 Prevention of type 2 Diabetes in subjects with prediabetes and meta- bolic syndrome treated with phentermine and topiramate extended release. Diabetes Care [epub ahead of print]

36. Margulis AV, Mitchell AA, Gilboa SM, et al, 2012 Use of topiramate in pregnancy and risk of oral clefts. Am J Obstet Gynecol 207: 405e1-7.

37. Green MW, Seeger JD, Peterson C, Bhattacharyya A, 2012 Utilization of topiramate during pregnancy and risk of birth defects. Headache 52: 1070-1084.

38. Hunt S, Russell A, Smithson WH, et al, 2008 Topiramate in pregnancy: preliminary experience from the UK Epilepsy and Pregnancy Register. Neurology 71: 272-276.

39. Malgarini RB, Pimpinella G, 2011 Phentermine plus topiramate in the treatment of obesity. Lancet 378 : 125-126.

40. Heal DJ, Gosden J, Smith SL, 2012 What is the prognosis for new centrally-acting anti-obesity drugs? Neuropharmacology 63: 132-146. 